\title{
Optically Tunable Surfaces with Trapped Particles in Microcavities
}

\author{
R. Sainidou and F. J. García de Abajo \\ Instituto de Óptica-CSIC, Serrano 121, 28006 Madrid, Spain \\ (Received 29 May 2008; revised manuscript received 17 July 2008; published 23 September 2008)

\begin{abstract}
We introduce optically tunable surfaces based upon metallic gold nanoparticles trapped in open, waterfilled gold cavities. The optical properties of the surfaces change dramatically with the presence and location of the particles inside the cavities. The precise position of the particles is shown to be controllable through optical forces exerted by external illumination, thus leading to all-optical tunability, whereby the optical response of the surfaces is tuned through externally applied light. We discuss the performance of the cavity-particle complex in detail and provide theoretical support for its application as a novel concept of a large-scale optically tunable system.
\end{abstract}

DOI: 10.1103/PhysRevLett.101.136802

PACS numbers: 73.20.Mf, 78.67.Bf, 78.68.+m

The last decade has witnessed tremendous progress in optical trapping and manipulation of small particles with a wide range of applications, ranging from physics and engineering to biology and medicine [1,2]. A fascinating possibility consists in controlling and tuning the optical response of nanoscale systems, thus opening the field to biosensing and all-optical switching. Early attempts to bind small particles using light forces [3-7] led to the development of optical tweezers [3,6], capable of trapping and aligning objects ranging from microorganisms [6] to metallic nanoparticles [8,9]. Manipulation of microparticles using plasmons has been recently demonstrated as well [10], whereas fine-tuning of nanoparticle positions has been theoretically proved to be realizable by coupling to plasmonic nanostructures [11].

In this Letter, we explore the optical tunability of cavitynanoparticle systems by considering an open microvoid in an otherwise flat gold surface surrounded by water and containing a gold nanorod in its interior. The nanoparticle is the key to purposely modify the optical response of the cavity-particle composite system. More precisely, we demonstrate through boundary-element-method [12] simulations the possibility of optically trapping the particle at positions that depend on the wavelength of externally incident light, thus producing tunable changes in the optical response of the system. The driving optical force can be used to displace the trapped particle at will inside the cavity, which results in changes of the surface reflectivity. Water is a typical solvent for nanoparticle dispersions, but our qualitative conclusions do not rely on the specific choice of this medium.

The optical properties of spherical nanocavities in planar gold surfaces are well understood from the experimental and theoretical points of view $[13,14]$, leading to such phenomena as omnidirectional total absorption of light [15]. We consider, in particular, a cavity of radius $R=$ $300 \mathrm{~nm}$ and depth $450 \mathrm{~nm}$ immersed in water, as shown in Fig. 1(a). The system is illuminated with $p$-polarized light incident at $45^{\circ}$ with respect to the flat gold surface. The cavity exhibits resonances associated with trapped modes [14], which appear as sharp peaks in the absorption spectrum [see Fig. 1(b)]. In particular, the pronounced resonance at $985 \mathrm{~nm}$ is associated with a near-field intensity distribution with an important localization at the center of the cavity [see inset of Fig. 1(b)], implying the possibility of strong interaction with the corresponding plasmon modes of any nanoparticle placed in that region. These nanoparticle plasmons can be tuned close to the cavity resonance wavelength. Actually, gold nanorods are good candidates for that purpose, since their longitudinal plasmon modes can be easily excited [16] and their wavelength depends strongly on the dimensions of the cylindrical rod, denoted here as $\left(\right.$ diameter $\left.=2 R_{p}\right) \times($ length $=L)$. An example is given in Fig. 1(c) for a $20 \mathrm{~nm} \times 100 \mathrm{~nm}$ gold nanorod. A longitudinal plasmon is clearly observed at $970 \mathrm{~nm}$.

When the particle is placed inside the cavity, the interaction between their respective plasmon resonances depends on the overlap between their associated fields. We assume for simplicity that the particle is aligned along the rotational axis of the cavity (the $z$ axis), and therefore strong overlap occurs for cavity modes localized at the center of a cavity such as that of Fig. 1(b). Let us first assume that the rod is in the center of the cavity (i.e., $210 \mathrm{~nm}$ away from the opening), close to the maximum of the cavity-mode intensity. The rod-cavity interaction gives rise to two distinct peaks in the absorption spectrum of the composite system that are displaced with respect to the noninteracting cavity and rod modes. For instance, we observe modes at wavelengths 910 and $1025 \mathrm{~nm}$ for the $20 \mathrm{~nm} \times 100 \mathrm{~nm}$ gold nanorod, far away from the initial resonances of the two individual constituents of the system, formerly at wavelengths of 970 (rod) and $985 \mathrm{~nm}$ (cavity), as shown in Fig. 1. The aspect ratio (AR) of the rod plays a crucial role for the wavelength position of these two resonances, as the longitudinal mode of the nanorod can be swapped over a wavelength interval around the cavity-mode wavelength for varying AR. This is illustrated 


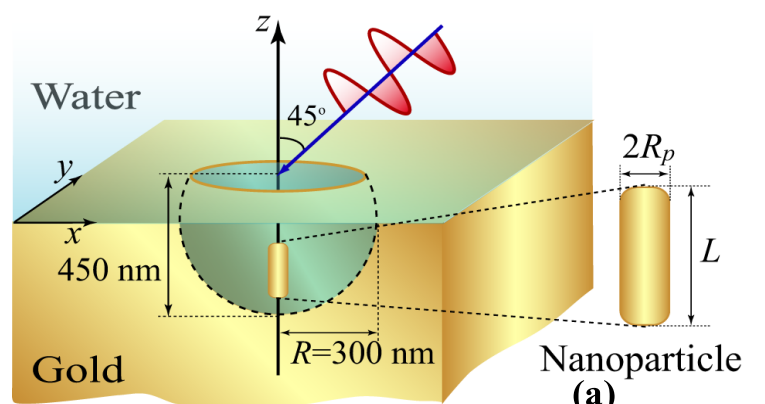

(a)

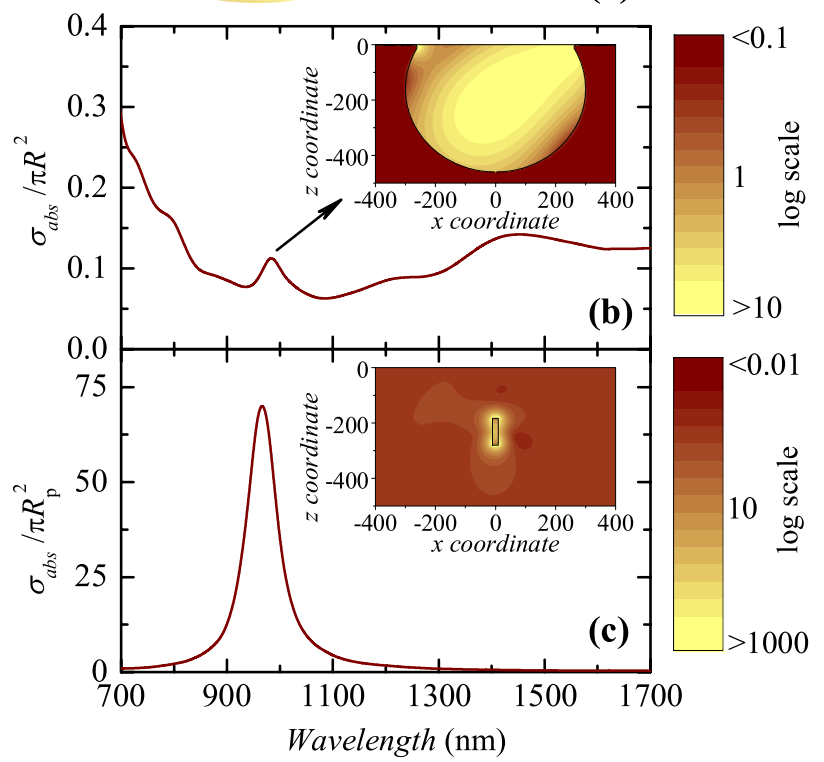

FIG. 1 (color online). (a) Cavity-particle system under study, including definitions of the cavity dimensions, and particle radius $R_{p}$ and length $L$. The cavity, excavated in an otherwise infinite planar gold-water interface, is illuminated by $p$-polarized (TM) light under $45^{\circ}$ incidence with respect to the planar surface. The gold nanorod is assumed to be placed in different positions along the cavity axis ( $z$ axis). (b), (c) Normalized absorption cross section $\sigma_{\text {abs }}$ of the single cavity and a single nanorod of dimensions $20 \mathrm{~nm} \times 100 \mathrm{~nm}$, respectively. Near-field-intensity plots at the resonance wavelengths 985 and $970 \mathrm{~nm}$ are shown in the insets.

in Fig. 2(a), where an avoided crossing is clearly discernible.

In a quantum-mechanical analogy, we can assume that the cavity-nanoparticle composite system is governed by an effective Schrödinger equation of the form $\hat{H}|\Phi\rangle=$ $\omega|\Phi\rangle$, with the Hamiltonian $\hat{H}=\hat{H}_{0}+\hat{V}$, where $\hat{H}_{0}$ is the operator describing the individual constituents of the system with eigenstates $\left|n_{1}\right\rangle$ (cavity mode) and $\left|n_{2}\right\rangle$ (rod mode) and corresponding eigenvalue frequencies $\omega_{1}$ and $\omega_{2}, \hat{V}$ describes the interaction between cavity and particle, and we can approximate the state of the system as $|\Phi\rangle=$ $a_{1}\left|n_{1}\right\rangle+a_{2}\left|n_{2}\right\rangle$, that is, the hybridization of cavity and particle modes with superposition coefficients $a_{1}$ and $a_{2}$, respectively. Defining the coupling parameter $U=$ $\left\langle n_{1}|\hat{V}| n_{2}\right\rangle=\left\langle n_{2}|\hat{V}| n_{1}\right\rangle$, we find that the two eigenfrequen-

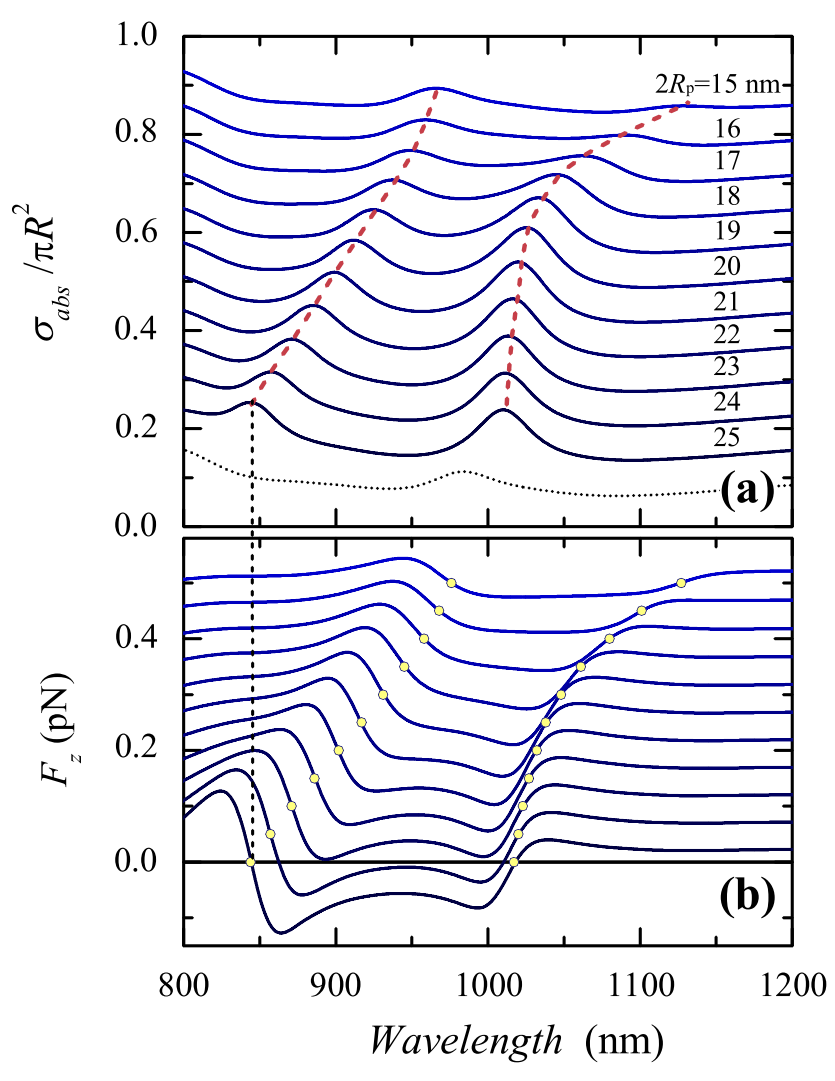

FIG. 2 (color online). (a) Absorption spectra of the rod-cavity composite system for different rod diameters $2 R_{p}$ (see labels) and fixed rod length $L=100 \mathrm{~nm}$. The rod is placed along the $z$ axis with its center $210 \mathrm{~nm}$ below the cavity opening. The spectra are vertically displaced for clarity. The dashed lines indicate the position of resonance maxima. The dotted curve shows the cavity without a particle. (b) Vertical force acting on the rod under the same conditions as in (a) for an external light intensity of $1 \mathrm{~mW} / \mu \mathrm{m}^{2}$. The different curves are displaced for clarity with the open symbols denoting the zero-force points.

cies $\omega_{ \pm}$of the composite system are given by $\omega_{ \pm}=$ $\left(\omega_{1}+\omega_{2}\right) / 2 \pm\left[\left(\omega_{1}-\omega_{2}\right)^{2} / 4+U^{2}\right]^{1 / 2}$. The hopping parameter $U=0.072 \mathrm{eV}$ is determined by fitting this expression to the values $\omega_{ \pm}$found numerically from the absorption spectra of Fig. 2(a), which are represented as solid curves in Fig. 3(a) and compared to this analytical expression (dashed curves).

The hybridization can be also observed in the near-field plots at the resonance wavelengths of the composite system. An example is given in Figs. 3(b) and 3(c) for the $16 \mathrm{~nm} \times 100 \mathrm{~nm}$ rod. The near-field plots clearly show a rodlike and a cavitylike profile at the corresponding resonance wavelengths of 960 and $1090 \mathrm{~nm}$ [cf. insets of Figs. 1(b) and 1(c)], since they are located close to the single-rod $(1080 \mathrm{~nm})$ and single-cavity $(985 \mathrm{~nm})$ modes, respectively. We intend to obtain significant changes in the resonance wavelength of the composite system triggered by the presence of the nanoparticle, and thus we need to 

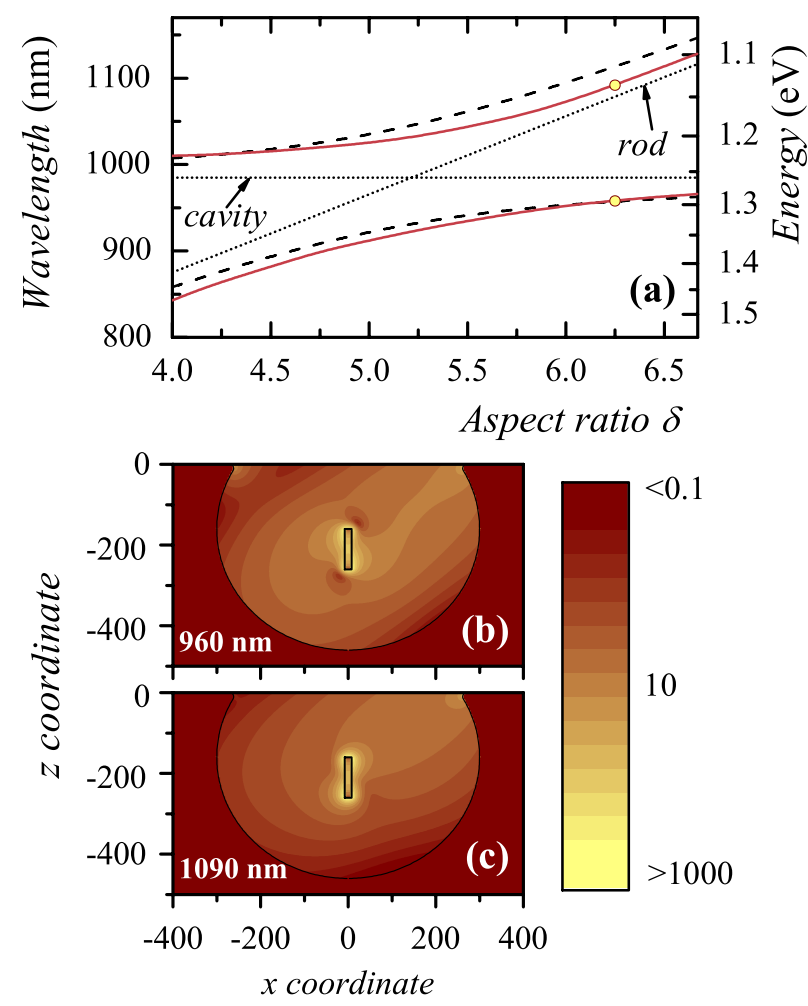

FIG. 3 (color online). (a) Resonance modes of the rod-cavity system extracted from Fig. 2(a) as a function of the particle aspect ratio $\delta=L / 2 R_{p}$ (solid curves). Dotted curves: singlecavity and single-rod resonance modes. Dashed curves: analytical-model approximation (see text). (b),(c) Near-fieldintensity plots for the $16 \mathrm{~nm} \times 100 \mathrm{~nm}$ rod [see open circles in (a)] at the resonance wavelengths 960 and $1090 \mathrm{~nm}$ of the composite system.

work close to the crossing point $\left(\omega_{1}=\omega_{2}\right)$ of the singlerod and single-cavity modes [i.e., with $\mathrm{AR} \delta \simeq 5.25$, according to Fig. 3(a)] in order to maximize mode repulsion, approximately given by $U$. Therefore, we will consider $20 \mathrm{~nm} \times 100 \mathrm{~nm}$ rods in what follows.

The observed change in the optical response of the cavity due to the presence of the nanorod in its interior cannot be realized without a tool to keep the nanoparticle trapped inside the cavity at a designated position. In fact, our system offers such a possibility as we show next. We calculate the optical force exerted on the particle for a given external illumination following the methods described in, for instance, Ref. [17]. Hereafter, we will be concerned only with the $z$-component force, $F_{z}$, assuming that its $x$ component is canceled out by adding a second external light beam propagating to the opposite $x$ direction with respect to the first one (i.e., the two beams are symmetrically placed with respect to the $y z$ plane). Incidentally, the cavity mode under consideration has maximum electric field near the center of the cavity, with dominant component along its rotation axis, thus facilitating the alignment of the rod. We represent in Fig. 2(b) the force acting on the nanorods considered in Fig. 2(a) for an external light intensity of $1 \mathrm{~mW} / \mu \mathrm{m}^{2}$. The magnitude of the force is large enough to overcome effects due to friction or Brownian motion, contributing with forces of a few $\mathrm{fN}$ [18]. Gravity forces are even much weaker. Also notice that the force is proportional to the light intensity, so that even much weaker incident illumination will produce sufficient trapping stability, particularly if one is concerned with the response of a statistically large number of cavities, as is the case in macroscopic surfaces [13]. Interestingly, the cavity mode has dominant evanescent character (the field is a few times larger inside the cavity compared to the incident field), implying maximum gradient forces right on resonance, accompanied by weak extinction forces, since evanescent waves do not propagate real momentum.

The absorption spectrum of the cavity-rod system is quite sensitive to changes in the position of the rod along the $z$ axis, as expected from the degree of overlap between the particle and the cavity-mode intensity [see Fig. 1(b)]. This is illustrated in Fig. 4(a). Our system is thus a good candidate to exhibit all-optical tunability: the use of optical forces exerted on the particle through external illumination can displace the nanorod along the $z$ axis to trigger changes in the absorption or reflection of the system. We show these forces in Fig. 4(b), calculated for an external light intensity of $1 \mathrm{~mW} / \mu \mathrm{m}^{2}$. Three different rod positions along the $z$ axis are considered $(60,230$, and $330 \mathrm{~nm}$ from the cavity aperture). For the first resonance mode located at lower wavelengths, the force is directed downwards when the particle lies at the deepest position (dotted curves), and it changes to upwards at less deep positions (dashed and solid

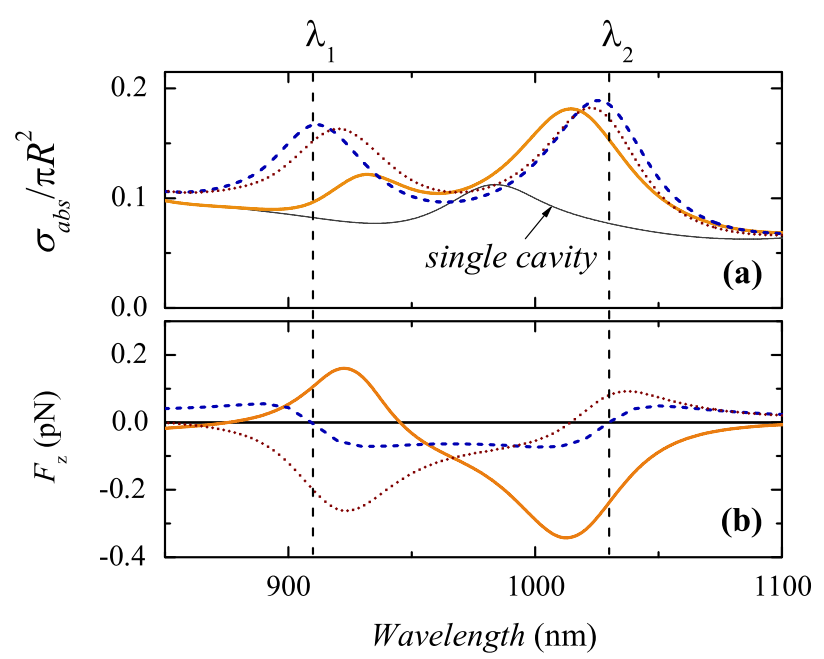

FIG. 4 (color online). (a) Absorption spectra of the rod-cavity system for a $20 \mathrm{~nm} \times 100 \mathrm{~nm}$ rod placed at different depths from the cavity opening: $-60,-230$, and $-330 \mathrm{~nm}$ (solid, dashed, and dotted curves, respectively). The single-cavity absorption is shown for reference. (b) Forces along the $z$ axis acting on the rod for the cases considered in (a). The vertical dashed lines are guides to the eye (see text). 


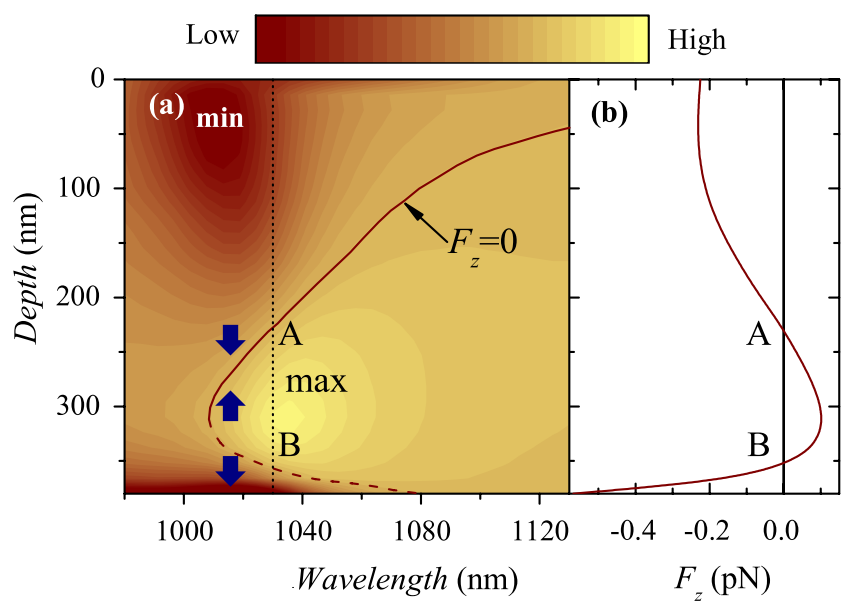

FIG. 5 (color online). (a) Vertical force acting on the rod as a function of light wavelength and particle position (depth) below the cavity opening. Solid and dashed curves denote stable and unstable equilibrium (zero-force) points, respectively. The thick arrows visualize the direction of the optical force in the vicinity of the wavelength $1030 \mathrm{~nm}$, indicated by the dotted vertical line, for which the detailed form of the force is presented in (b). Points $A$ and $B$ show stable and unstable trapping positions inside the cavity, respectively.

curves). Considering, for example, an operating wavelength $\lambda_{1}=910 \mathrm{~nm}$, the optical force can remove the particle from the cavity, thus changing the absorption of the composite system from 0.150 to 0.095 (see left vertical dashed line in Fig. 4). The opposite happens for the second resonance mode located at higher wavelengths: the force is directed downwards when the rod is placed close to the top of the cavity (solid curves), thus pushing the particle downwards. For an operating wavelength $\lambda_{2}=1030 \mathrm{~nm}$ (see right vertical dashed line in Fig. 4) the trapping of the particle becomes possible inside the cavity.

This is demonstrated in more detail in Fig. 5. The $z$ component of the force acting on the rod placed at different depths inside the cavity is shown for different wavelengths in Fig. 5(a) (light intensity equal to $1 \mathrm{~mW} / \mu \mathrm{m}^{2}$ ). The force surface map exhibits stable and unstable equilibrium (zero-force) points, denoted by solid and dashed curves in the figure, respectively. Stable trapping of the rod at a given depth inside the cavity occurs when the force changes direction from positive to negative when moving from below to above that depth. Consequently, this will be the case for wavelengths in the 1020-1040 nm range and for points lying on the stable-equilibrium line [solid curve in Fig. 5(a)]. As an example, we choose the wavelength of $1030 \mathrm{~nm}$. The corresponding depth-profile force is depicted in Fig. 5(b). For this specific wavelength, point $A$ (depth $\sim 220 \mathrm{~nm}$ ) represents a trapping position for the rod, while point $B$ (depth $\sim 350 \mathrm{~nm})$ is an antitrapping position.

In conclusion, we have shown that composite systems formed by gold nanoparticles trapped inside water-filled gold nanocavities exhibit large variations in optical absorption as a function of the position of the particle inside the cavity. Optical forces acting on the particle have been shown to be sufficiently large to move the particles around, and in particular positions and illumination wavelengths have been identified that lead to stable particle trapping. The combined cavity-nanoparticle system is thus tunable via the wavelength of a pump light source, which triggers dramatic changes in the reflectance spectrum experienced by a second probing light source. Our findings are thus opening a new path towards all-optical switching.

We thank Romain Quidant for helpful discussions. This work was supported by the Spanish MEC (NAN200408843-C05-05 and MAT2007-66050) and by the EU-FP6 (NMP4-2006-016881 “SPANS”).

[1] D. G. Grier, Nature (London) 424, 810 (2003).

[2] A. Ashkin, Proc. Natl. Acad. Sci. U.S.A. 94, 4853 (1997).

[3] A. Ashkin, Phys. Rev. Lett. 24, 156 (1970).

[4] C. A. Ashley and S. Doniach, Phys. Rev. B 11, 1279 (1975).

[5] A. Ashkin, Science 210, 1081 (1980).

[6] A. Ashkin and J. M. Dziedzic, Science 235, 1517 (1987).

[7] M. M. Burns et al., Phys. Rev. Lett. 63, 1233 (1989); Science 249, 749 (1990).

[8] P. M. Hansen et al., Nano Lett. 5, 1937 (2005).

[9] M. Pelton et al., Opt. Lett. 31, 2075 (2006); M. Righini et al., Phys. Rev. Lett. 100, 186804 (2008).

[10] M. Righini et al., Nature Phys. 3, 477 (2007); R. Quidant and C. Girard, Laser Photonics Rev. 2, 47 (2008).

[11] L. Novotny et al., Phys. Rev. Lett. 79, 645 (1997); F. J. García de Abajo et al., J. Phys. B 40, S249 (2007).

[12] F. J. García de Abajo and A. Howie, Phys. Rev. Lett. 80, 5180 (1998); Phys. Rev. B 65, 115418 (2002).

[13] S. Coyle et al., Phys. Rev. Lett. 87, 176801 (2001).

[14] R. M. Cole et al., Nano Lett. 7, 2094 (2007).

[15] T. V. Teperik et al., Nat. Photon. 2, 299 (2008).

[16] C.F. Bohren and D. R. Huffman, Absorption and Scattering of Light by Small Particles (WileyInterscience, New York, 1983).

[17] F. J. García de Abajo, J. Quant. Spectrosc. Radiat. Transfer 89, 3 (2004).

[18] At room temperature $T \sim 300 \mathrm{~K}$ and for distances $D \sim$ $100 \mathrm{~nm}$ (i.e., of the order of the cavity size), the thermal force can be roughly estimated from $F_{\text {th }} \sim K_{B} T / D$ to be of the order of $40 \mathrm{fN}$, where $K_{B}$ is the Boltzman constant. Heating by the incident light produces only a small relative increase in temperature because gold is poorly absorbing and has large thermal conductivity. 